\title{
Pembrolizumab in the Treatment of Metastatic Gastric Cancer
}

\section{Rakhimov R Radmir ${ }^{1 *}$, AA Izmailov ${ }^{1}$, ON Lipatov ${ }^{1,2}$, AV Sultanbaev ${ }^{1}$, KV Menshikov ${ }^{1,2}$, AF Nasretdinov ${ }^{1}$ and VA Surovyatkin ${ }^{1}$}

${ }^{1}$ State Autonomous Institution of Healthcare Republican Clinical Oncological Dispensary of the Ministry of Health of the Republic of Bashkortostan, Ufa, Russia ${ }^{2}$ Federal State Budgetary Educational Institution of Higher Education "Bashkir State Medical University" of the Ministry of Health of the Russian Federation, Russia

*Corresponding Author: Rakhimov R Radmir, State Autonomous Institution of Healthcare Republican Clinical Oncological Dispensary of the Ministry of Health of the Republic of Bashkortostan, Ufa, Russia.
Received: September 02, 2021

Published: September 20, 2021

(C) All rights are reserved by Rakhimov $\mathbf{R}$

Radmir.

\section{Abstract}

The incidence of stomach cancer has been declining over the past decade, but unfortunately it is still the fifth most common disease with the third death rate among cancers. Diagnosis of stomach cancer usually occurs at the stage of neglect and incurability (stages III - IV), in the early stages (I - II stages) clear symptoms do not appear. $25 \%$ of patients have advanced gastric cancer, the other 25 - 50\% progress to metastatic gastric cancer. The prognosis is especially poor for patients who have not responded to 1 line of chemotherapy. In the United States in 2012, 54.5\% of patients received second and third lines. Five-year survival rate is $30 \%$ among all stages. In recent years, new drugs have emerged in the treatment of stomach cancer that needs to be studied. Pembrolizumab demonstrated efficacy in PD-L1-positive advanced gastric/gastroesophageal junction cancer in the first-, second-, and third-line setting in KEYNOTE-062, KEYNOTE-061, and KEYNOTE-059, respectively.

In KEYNOTE-062, median follow-up was 11 months, median OS (pembrolizumab vs. chemotherapy) was 17 months versus 11 months (HR, 0.69; 95\% CI, 0.49-0.97), median PFS was 3 months versus 6 months (HR, 1.09, 95\% CI; 0.79-1.49), 0RR was 25\% versus $38 \%$, and median (range) DOR was 19 months (1+ to $34+$ ) versus 7 months (2+ to $30+$ ).

Keywords: Pembrolizumab; Gastric Cancer; Immune Therapy; Checkpoint Inhibitor; Systemic Therapy

\section{Introduction}

Stomach cancer and esophageal cancer occupy the third and sixth places among the causes of death from cancer. In 2017, 28,000 new cases of stomach cancer were registered in the United States and 10,960 people died from this disease. In the same year, 16,940 new cases were detected and 15,690 patients died from esophageal cancer. While the number of new cases of esophageal squamous cell carcinoma and distal gastric adenocarcinoma is decreasing in the United States, the frequency of new patients with proximal esophageal-gastric junction adenocarcinoma is increasing, including Sievert I, II, III. This is due to an increase in obesity in the population and esophageal-gastric reflux [1,2,35].

Pembrolizumab, known as MK-3475 and lambrolizumab - is a human monoclonal IgG4 kappa antibody with an approximate mass of $149 \mathrm{kDa}$, preventing the connection of PD-1 with PD-L1 and PDL2. The mechanism of action. The PD-1 transmembrane receptor is present on a variety of immune cells, including $\mathrm{T}$ cells, and is a control point that modulates the immune response. The addition of PD-1 with the PD-L1 and PD-L2 ligands leads to the transduction of inhibitory signals that reduce the regulation of $\mathrm{T}$ cells, reducing the ability of T cells to destroy neoplastic cells $[3,4,37,38]$. PD-L1 is 
a transmembrane protein on the surface of some tissues, including neoplastic cells. Cancer cells can be destroyed by T cell immunity and PD-L2 expression by PD-L1 expression together with inhibition of the signaling pathway. Pembrolizumab binds to PD-1 and blocks the binding to PD-L1 and PD-L2 by removing the physiological brake, activating the immune system and restoring the antitumor response [5,6,36].

PD-L1 expression has been studied in gastric cancer, but the prognosis of patients ' life is clear. In 11 studies, PD-L1 expression was found to be a negative prognostic factor for overall survival. However, in 3 studies, this biomarker was evaluated as a positive prognostic factor, moreover, in one study, no correlation was found between PD-L1 expression and overall survival $[7,8]$.
The combined positive score (CPS) is the number of PD-L1 positive cells (cancer cells, lymphocytes, macrophages) divided by the total number of living cancer cells multiplied by 100. PD-L1 expression is significantly associated with MSI, the presence of EpsteinBarr virus and Helicobacter pylori with a large proportion of PD-L1 CPS $\geq 1$ tumors with MSI high, positive tests for Epstein-Barr virus and Helicobacter pylori. There is no significant association between PD-L1 expression and amplification of the HER 2 gene $[9,10,39]$. The level of PD-L1 mRNA is moderately correlated with CPS. PD-L1 GPS was moderately correlated with two mRNA signatures, which led to quantitative measurement of immune activity in the tumor microenvironment: CYT and evaluation of gene profile expression (GEP) (Figure 1 and 2) [11,15,42].
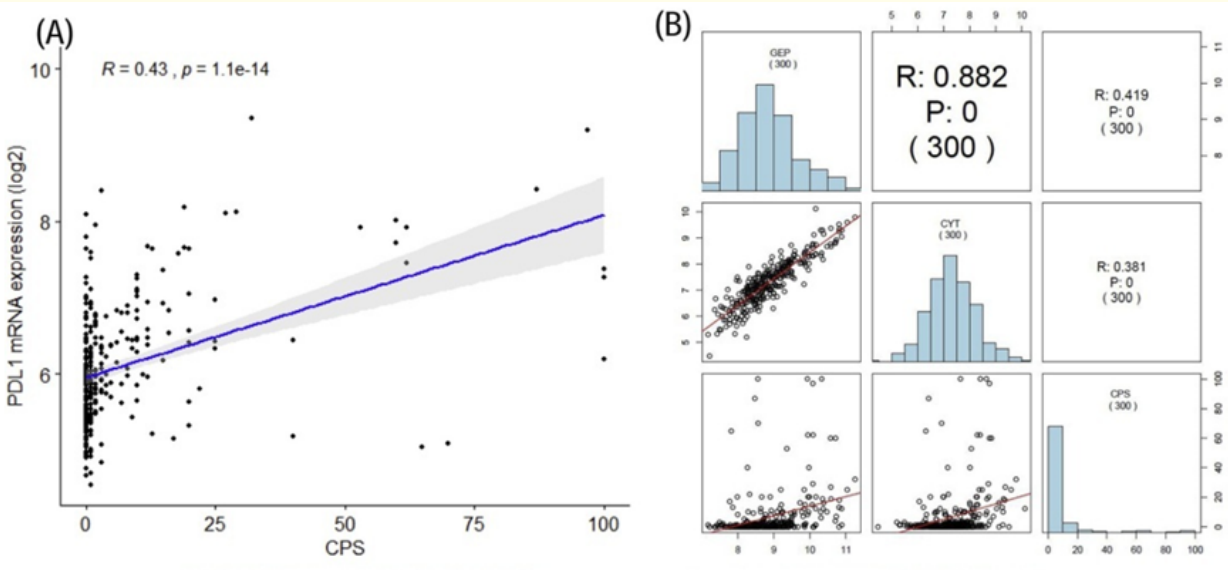

Figure 1: Association between PD-L1 expression and PD-L1 mRNA, CYT and evaluation of gene profile expression (GAP) using CPS.
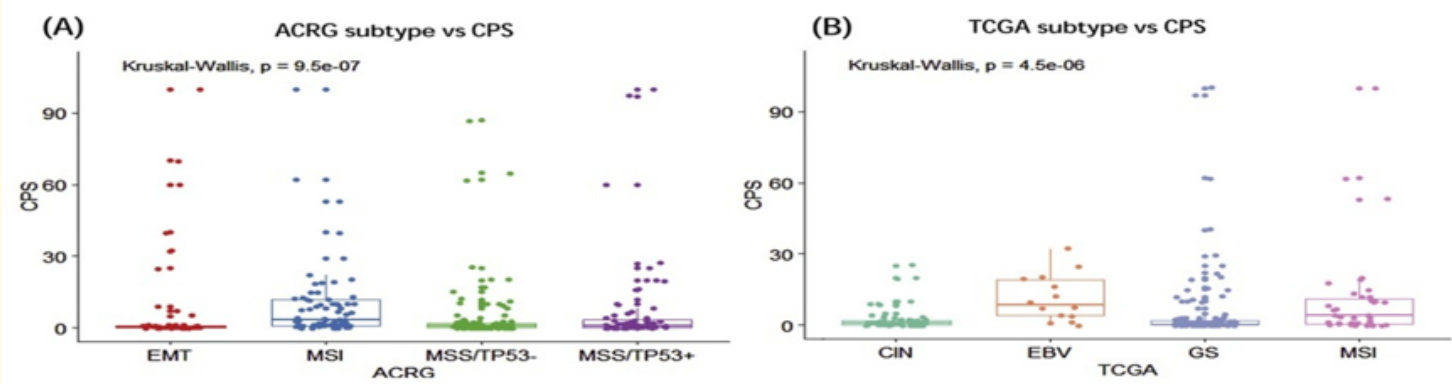

Figure 2: A molecular subtype of the Asian Cancer Research Group (AACR) and B molecular subtype of the Cancer Genomic Atlas (TCGA). CIN, chromosomal instability, CYT, cytolytic activity, GEP, gene expression profile, GS, genomic instability, EBV, Epstein-Barr virus, EMT, epithelial mesenchymal transition, MSI, microsatellite instability, MSS, microsatellite stability. 
PD-L1 expression is associated with the molecular subtype of the Asian Cancer Research Group with high CPS in the MSI subgroup. PD-L1 expression was also associated with the molecular subtype of the cancer genomic atlas with high CPS in the MSI and Epstein-Barr virus subgroups [12,15,41,42].
Overall survival was greater in patients with PD-L1 CPS $\geq 1$ than in patients with PD-L1 CPS $<1$. The median overall survival was not achieved in the first case, it was 40 months in the second case. In the case of localized gastric cancer, PD-L1 GPS = 1 is associated with a long overall survival compared to PD-L1 negative tumors (Figure 3-5) [13,15].

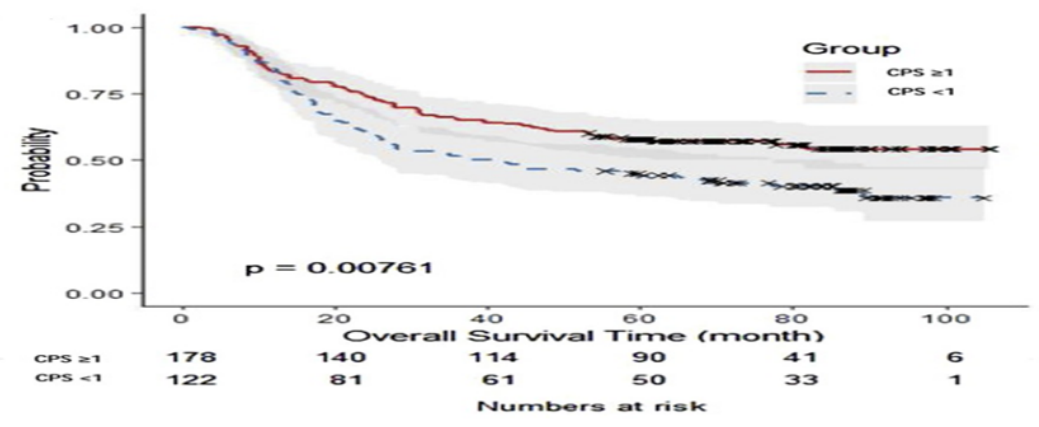

Figure 3: Overall survival in gastric cancer.

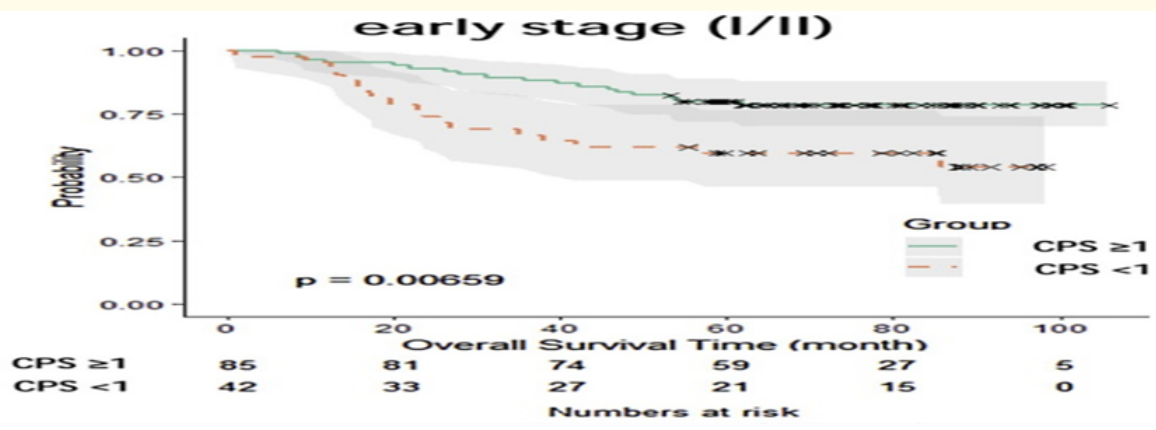

Figure 4: Overall survival in localized gastric cancer.

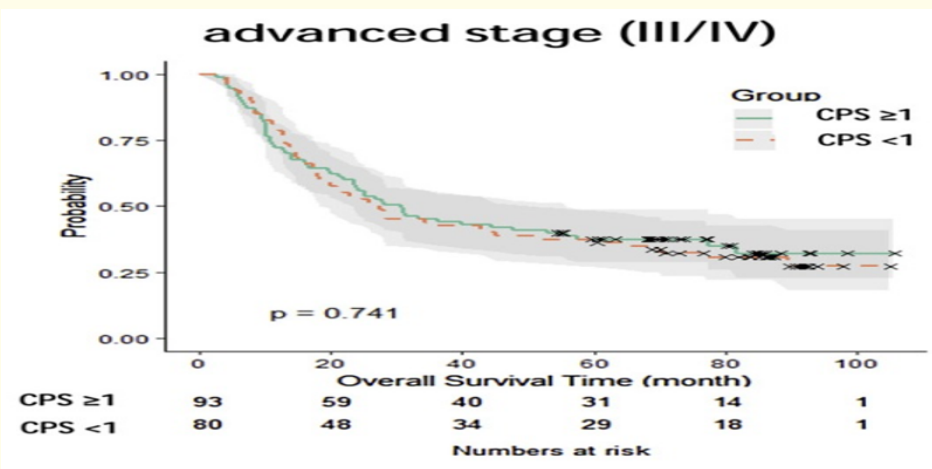

Figure 5: Overall survival in advanced gastric cancer. 
A high PD-L1 CPS score can be a predictor of a good prognosis of patients ' life, including for anti-PD-1/PD-L1 therapy. However, it is necessary to obtain more data from other ongoing studies in order to come to a correct conclusion $[14,15,43]$.

Japanese scientist Yuji Eso and co-authors described the path of the appearance of microsatellite instability. Among the various DNA repair pathways, the MMR pathway plays a key role in maintaining DNA replication and genome stability. MMR maintains genomic integrity by correcting DNA mismatch base replacement, frame shift (insert/delete), and slippage. These are conditions that are caused by DNA replication errors. In eukaryotes, MMR recognizes mismatches in two protein complexes: MutSa (the heterodimer of MutS homologue 2 [MSH2] and the proteins MutS homologue 6 [MSH6]) and MutSb (the heterodimer of MSH2 and the MutS homologue 3 [MSH3] proteins) [16,18,44]. MutSa recognizes mismatches with base replacement and small (up to 3 nucleotides) insertion or deletion loops, while MutSb recognizes larger insertion or deletion loops up to 13 nucleotides in size and does not restore base substitution. MutSa or MutSb binds to an erroneous pairing in an adenosine triphosphate-dependent manner, and subsequently recruits MutLa (a heterodimer of MutL homologue 1 [MLH1] and a post-meiotic segregated enlarged 2 [PMS2] protein). MutLa forms a triple complex with MutS when mismatched. Proliferation of the cell's nuclear antigen activates latent endonuclease in the PMS2 subunit of MutLa, which makes the DNA break from 5 ' to a mismatch. After the DNA incision step, exonuclease 1 is recruited and activated by MSH2 and/or MLH1. Activated exonuclease 1 catalyzes the removal of the nascent DNA chain before and slightly above the mismatch. The cut-out gap of DNA is resynthesized by polymerase $d$ stimulated by the nuclear antigen of proliferating cells, and the remaining gap is closed by DNA ligase I. Since the MMR pathway described above plays an important role in maintaining DNA accuracy by correcting DNA replication errors, therefore, MMR deficiency leads to additive mutations throughout the genome and a strong hypermutatory phenotype known as MSI (Figure 6) $[17,19,45,46]$.

Among the human DNA sequences, there are more than 100,000 sections of short tandem repeating sequences, called microsatellites, which are particularly sensitive to detecting the MMR path error. Cells with an abnormally functioning MMR pathway cannot correct errors during DNA replication, which causes the creation of an inappropriate number of microsatellite nucleotide repeats, which leads to instability of microsatellite regions (Figure 7) $[20,47]$.

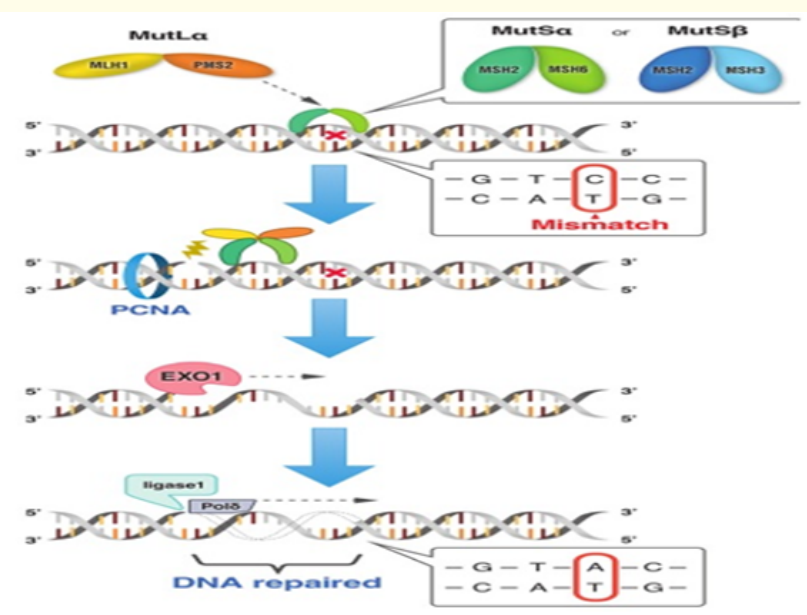

Figure 6: Schematic diagram of the DNA mismatch recovery path.

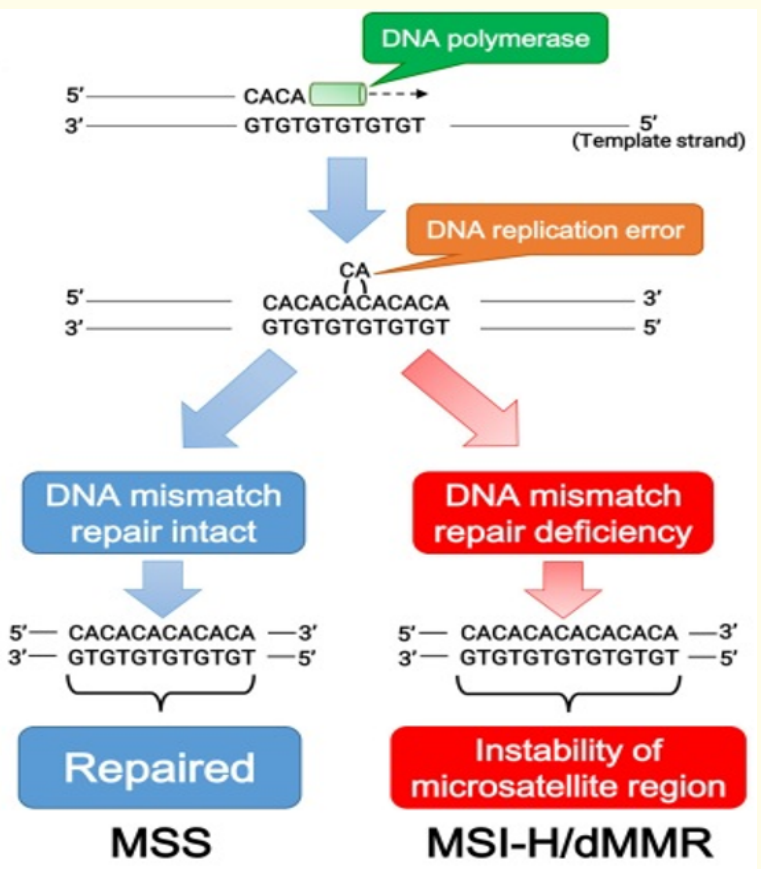

Figure 7: Schematic diagram of microsatellite stability and microsatellite instability of a high degree or insufficiency of mismatch recovery. 
MSI reflects the state of genetic hypermutability resulting from a violation of the MMR of DNA, which is accompanied by an increase in the frequency of mutations by 100 - 1000 times. The presence of MSI is a sign of sporadic or hereditary dysfunction of the MMR pathway caused by various factors, including mutations in genes associated with MMR, inactivation of the MMR gene transcription due to hypermethylation of its promoter region, or inhibition of transcription caused by inflammation $[21,48]$.

Benjamin A. Weinberg and colleagues published the results of a study on immune biomarkers for stomach cancer and cardioesophageal cancer. In their studies of 581 samples of gastric adenocarcinoma and esophageal-gastric junction, a high tumor mutation load and the status of MSI-H were identified in 30 patients $(5.2 \%)$ with PD-L1 negative tumors with a threshold value of $1+, 1 \%[22,25,49]$. Immunotherapy could be useful for these patients, but pembrolizumab therapy was excluded based on the FDA decision. Using a higher threshold of 2+, 5\% PD-L1, an additional 34 patients had a high tumor mutation load and MSI-H status. Immunotherapy could also be useful for these patients. Based on the performed gene sequencing of a new generation, a rare tumor mutation POLE was identified, which is functionally equivalent to MSI-H and responds well to treatment with checkpoint inhibitors [23]. They noted that primary tumors had a high frequency of tumor mutation load and MSI-H, while in unpaired metastases, the indicators of the average tumor mutation load were similar. PD-L1 expression was similar in primary and metastatic tumors. The low frequency of high tumor mutation load and MSI-H in metastases may indicate intra-tumor heterogeneity with early clonal divergence. It is necessary to conduct studies with large cohorts with paired and metastatic samples to confirm the above hypothesis. In conclusion, they believe that the PD-L1 expression test may not adequately identify patients who would benefit from immunotherapy. The addition of a tumor mutation load to PD-L1 expression should be added in future clinical studies. A more routine use of next-generation gene sequencing can help practicing oncologists better select patients with gastric adenocarcinoma and cardioesophageal transition for treatment with immune checkpoint inhibitors by evaluating the tumor mutation load and MSI [24].

R. Sundar and co-authors suggested that metastatic gastric cancer with high use of an alternative promoter would be resistant to anti-PD-1 therapy. They confirmed that there is a relationship between the use of an alternative promoter and intra-tumor immunity in advanced stomach cancer. Tumors with a high level of use of alternative promoters and, consequently, with lower predicted immunogenicity are more resistant to checkpoint inhibitors. Thus, the increased use of an alternative promoter may represent a new biomarker of the response to checkpoint inhibitors in metastatic gastric cancer $[25,26,50]$.

The group with low use of an alternative promoter demonstrated significantly increased expression of CD8A, GZMA and PRF1 when compared with the group with high use of an alternative promoter. The frequency of objective responses, defined as a partial or complete response to therapy, was higher in the group with low use of an alternative promoter than in the group with high use of an alternative promoter. (10/24 vs. $1 / 13, \mathrm{P} 0.03)$. Note, in the group with high use of an alternative promoter, the only response was in the MSI tumor subtype. The median disease-free survival was 55 days in the group with high use of an alternative promoter compared to 180 days in the group with low use of an alternative promoter ( $\log$ rank $\mathrm{P}=0.0076)$. In the group with low use of an alternative promoter, there were $17 \%$ of cases of Epstein-Barr virus and $12 \%$ of MSI high samples of the TCGA subtype, while the group with high use of an alternative promoter had only $8 \%$ of MSI and no samples of Epstein-Barr virus $[27,28]$. The relapse-free survival between different subtypes of TCGA was different (P 0.0026), while the subtypes of MSI and EBV have significantly longer survival [491 days (MSI/Epstein-Barr virus) compared to 80 days (chromosomal instability/genomic instability). It is noteworthy that among the subtype of chromosomal instability / genomic instability, relapsefree survival also significantly differed between groups with low and high use of an alternative promoter 48 days versus 161 days. The overall survival data were not ready at the time of publication of this article, but there is a tendency to increase survival in the group with low use of an alternative promoter (340 vs. 292 days, $\mathrm{P}=0.16$ ). Multivariate analysis of clinical and pathological use and use of alternative promoters showed that a high level of use of alternative promoters is an independent prognostic factor for relapse-free survival during treatment with pembrolizumab [HR $0.29,95 \%$ CI $0.099-0.85, \mathrm{P}=0.024)[29,30]$.

Sanjay V. Menghani and co-authors described a clinical case of cardia cancer with metastasis to the scalp. A 69-year-old patient with a history of osteoarthritis and sarcoidosis turned to an oncologist with complaints of difficulty swallowing and weight loss of 18 pounds in 2 months. He denied gastroesophageal disease and the presence of Helicobacter pylori. He underwent a PET/CT scan of the body. 
cT3N0Mx staging was performed. Histology: moderately differentiated adenocarcinoma. The patient was offered perioperative chemotherapy. However, the patient was treated in Mexico and China, where he received various methods of treatment. After 20 months from the diagnosis, a tumor was detected in the right occipital region, which bothered the patient. The skin over the tumor was erythematous and tense on palpation. After a biopsy of the tumor of the right occipital region, metastasis of stomach cancer was confirmed. Immunohistochemistry. Cytokeratin 7, cytokeratin 20, and REA were positive. The tumor was negative for the expression of $\mathrm{P}$ 63, thyroid transcription factor 1 and prostate-specific antigen. Repeated PET/CT of the body revealed foci in the liver, skeletal bones, and occipital region. After analyzing the tumor of the right occipital region, CARIS Molecular Intelligence PD-L1 was positive. The patient was prescribed pembrolizumab. Unfortunately, after several weeks of treatment, he died. Metastases to the skin of stomach cancer were described after 3-10 years from the initial diagnosis. Metastasis to the skin is the progression of gastric cancer in individual patients. Several similar clinical cases are described in the PubMed system. Lifshitz, Woo, and Cho presented metastases to the scalp or skin as a recurrence of stomach cancer. Sakaki found in 1979 metastases in the scalp and the dura mater of the right occipital region at an autopsy. Histology: gastric adenocarcinoma. Several clinical cases of neoplastic allopecia have been documented as metastatic paraneoplastic rashes of stomach cancer. Cutaneous metastases are rare, and clinically manifest manifestations of common visceral carcinoma, such as gastric adenocarcinoma. This clinical case highlights the need for examination of the skin in patients with a history of stomach cancer. The appearance of a tumor on the scalp can be an indicator of widespread visceral carcinoma, which can help in early diagnosis and improve the patient's treatment [31].

Gagandeep Brar conducted a comparative analysis of clinical studies on the effect of pembrolizumab on stomach cancer. The following results were obtained in the Phase $1 \mathrm{~B}$ clinical trial of KEYNOTE-012. The frequency of objective responses was $22 \%$. The MSI-H status was in $17 \%$ of patients, of which $50 \%$ achieved an objective response. $13 \%$ of patients had grade 3 and 4 side effects in the form of weakness, hypothyroidism, peripheral sensory neuropathy and pneumonitis. There were no fatal outcomes associated with treatment.

The study of the $2^{\text {nd }}$ phase of KEYNOTE-059. All patients received pembrolizumab $200 \mathrm{mg}$ intravenously every three weeks.
$57.1 \%$ of patients had a positive PD-L1 status, $42.9 \%$ of patients had a negative one. The frequency of objective responses was $15.5 \%$ in the group with a positive PD-L1 status and $6.4 \%$ in the group with a negative PD-L1 status. The average response time was 16.3 months in patients with positive PD-L1 and 6.9 months in patients with negative PD-L1. Surprisingly, 6 patients had a complete response to the treatment, including 3 patients with PD-L1 negative tumors. The frequency of objective responses in patients with MSI-H status was $57.1 \%$. The median disease-free survival was 2 months, the median overall survival was 5.6 months. In $17.8 \%$ of cases, there were side effects of grade 3 or higher, including two treatment-related deaths.

The phase 2 clinical trial of KEYNOTE-180 was aimed at studying pembrolizumab in a single mode in patients with metastatic cardioesophageal cancer and squamous cell carcinoma of the esophagus. The frequency of objective responses for the group with adenocarcinoma was $5.2 \%$ and $14.3 \%$ for the group of patients with squamous cell carcinoma of the esophagus. In the group of patients with a positive PD-L1 status, the frequency of objective responses increased to $13.8 \%$ and the frequency of disease stabilization was $36.2 \%$. However, the frequency of objective responses in the group with PD-L1 negative tumors was 6.3\%. The median disease-free survival was 2 months. The median overall survival was 5.8 months. In $12.4 \%$ of cases, side effects were observed, including one fatal outcome caused by pneumonitis.

Pembrolizumab was also studied in a phase 3 clinical trial. In this study, the superiority of pembrolizumab over paclitaxel was not proven. The PD-L1 CPS $\geq 1$ score was approximately $67 \%$. The median overall survival was 9.1 months in the pembrolizumab group and 8.3 months in the paclitaxel group. The median diseasefree survival was 1.5 months in the main group and 4.1 months in the control group. However, in the group that received pembrolizumab, a longer response to treatment was registered. The median average response in the main group was 18 months, when this indicator was 5.2 months in the control group. In 14\% of cases, side effects of grade 3 or higher were found in the group with pembrolizumab and in $35 \%$ of patients, these complications were detected in the group with paclitaxel. Despite the lack of superiority of pembrolizumab over paclitaxel, pembrolizumab showed an effective and long-lasting response to treatment in patients with a PD-L1 CPS score of $\geq 1$ with greater safety.

Citation: Rakhimov R Radmir., et al. "Pembrolizumab in the Treatment of Metastatic Gastric Cancer". Acta Scientific Gastrointestinal Disorders 4.10 (2021): 20-31. 
The clinical study KEYNOTE-059 is devoted to the study of the safety of immunotherapy and chemotherapy for stomach cancer. First-line patients with advanced gastric cancer and cardioesophageal cancer received pembrolizumab $200 \mathrm{mg}$ intravenously every 3 weeks with cisplatin and fluorouracil or capecitabine. PD-L1 expression was found in $64 \%$. The frequency of objective responses was at the level of $60 \%$ with $32 \%$ of patients with disease stabilization. The frequency of objective responses in the group with chemotherapy alone was 35\%. The average response period was 4.6 months. Side effects of the $3^{\text {rd }}$ and higher degree were in $75 \%$. No treatment-related deaths have been reported.

In 2019, the results of the clinical trial KEYNOTE-062 were presented at the meeting of the American Society of Clinical Oncology. Pembrolizumab and the combination of pembrolizumab with chemotherapy did not show superiority over chemotherapy. In patients with CPS $\geqslant 10$, the overall survival was 7 months. The median relapse-free survival in patients with CPS $\geqslant 1$ was 2 months in the group with pembrolizumab and 6.4 months this indicator was in the group with chemotherapy. Moreover, in patients with CPS $\geqslant 10$, chemotherapy appeared with a median overall survival of 6.1 months compared to 2.9 months in the group with pembrolizumab. Based on the above-described results of clinical studies, it can be concluded that future studies are needed to determine the optimal chemotherapeutic and immunotherapy combinations [32].

Rutika Mehta and co-authors described the cost-effectiveness of treatment with pembrolizumab. The clinical study KEYNOTE-045 showed the cost of treatment with pembrolizumab for 1 year, which amounted to 122,557 US dollars, which is higher than the price in other developed countries. Pembrolizumab therapy is considered cost-effective only in the United States, due to a significantly higher threshold of willingness to pay. Currently, clinical studies are underway aimed at studying pembrolizumab with chemotherapy drugs as a neoadjuvate drug therapy, for example, 03064490 (PROCEED), 02730546. The perioperative regime is studied in studies 02918162, 03488667, 03221426 (KEYNOTE-585), 03257163. Moreover, studies of palliative drug therapy in the first line for gastric cancer in various combinations of pembrolizumab with targeted and chemotherapy drugs are continuing, for example, 02494583 (KEYNOTE-062), 03342937 (KeyLargo), 02954536 [33].

Ian Chau and colleagues published the results of a phase $1 \mathrm{a} / \mathrm{b}$ clinical trial of JVDF, which was conducted in 11 medical centers in the United States, Great Britain, France, Spain and Germany. The median age was 63 years. 9 patients $(68 \%)$ had PD-L1 positive tumors. 6 patients $(21 \%)$ had PD-L1 negative tumors. The median therapy with ramucirumab and pembrolizumab was 4.5 months. A decrease in the dose of ramucirumab occurred in 2 (7\%) patients, while 19 (68\%) patients experienced a timing violation. Eleven (39\%) patients were forced to delay the administration of the dose of pembrolizumab (dose reduction was not allowed). The frequency of objective responses was $25 \%$. The time to respond to treatment was 2.7 months. The frequency of objective responses for PD-L1 positive tumors was $32 \%$ and $40 \%$ for CPS $\geq 10$ tumors. However, the frequency of objective responses for PD-L1 negative tumors was $17 \%$. The frequency of disease control was at the level of $67 \%$ for PD-L1 negative tumors, $68 \%$ for PD-L1 positive tumors and $80 \%$ for CPS $\geq 10$ tumors. The median relapse-free overall survival was 5.6 months. The median overall survival was 14.6 months. Patients with PD-L1 positive tumors had a greater relapsefree survival compared to PD-L1 negative tumors (8.6 months vs. 4.3 months), the same picture was with the median overall survival (17.3 months vs. 11.3 months). The median overall survival for $\mathrm{CPS} \geq 10$ tumors was 24.7 months [34].

\section{Clinical case from practice}

Patient F. R. V., born in 1946, fell ill in May 2018. She underwent anti-ulcer therapy, after the control FGDS, there was a negative dynamics. A blood transfusion was performed. On 27.12.2018, diagnostic laparoscopy was performed. On CT OBP, the thickening of the walls of the outlet part of the stomach to $13 \mathrm{~mm}$ for $95 \mathrm{~mm}$ with a narrowing of the lumen. Regional lymph nodes are not enlarged. Fibrogastroduodenoscopy (FGDS) On a small curvature from the upper third of the body to the pylorus is an extensive ulcerative defect with a flat bottom, high overhanging edges. The gatekeeper is passing through. Conclusion: BL of the stomach, ulcerative form, subtotal lesion.

She underwent 4 courses of neoadjuvate chemotherapy as a stage of perioperative chemotherapeutic treatment in the mode of Fluorouracil $2600 \mathrm{mg} / \mathrm{m}^{2}$ 24-hour infusion on day $1+$ oxaliplatin $85 \mathrm{mg} / \mathrm{m}^{2}$ on day $1+$ calcium folinate $200 \mathrm{mg} / \mathrm{m}^{2}$ on day 1 + docetaxel $50 \mathrm{mg} / \mathrm{m}^{2}$ on day 1 ; a cycle of 14 days. According to the FGDS of 28.02.2019 there is an ulcerated tumor in the antrum along the small curvature from the angle of the stomach to the pylorus. In the prepyloric department, ulceration is circular. The gatekeeper is narrowed, we pass. Conclusion: cancer of the output part of the stomach, infiltrative-ulcerative form. I passed a PET/CT scan 
of the body on 27.03.2019. In the outlet part of the stomach and in the bulb of the duodenum, a circular uneven thickening of the walls is determined, up to $20 \mathrm{~mm}$ with hyperfixation of FDG SUV max 12.83. There is an invasion of the perigastric fiber. The described walls are intimately attached and do not separate from the liver capsule at the S6 level, from the medial contour of the head of the pancreas and from the gallbladder (invasion). In the perigastric tissue, at the level of the described changes in the course of the hepatic-duodenal ligament, enlarged lymph nodes up to 11-12 mm are determined. Conclusion: According to PET/CT, there is a specific lesion of the output part of the stomach and the duodenal bulb, and regional lymph nodes and lymph nodes along the hepatic-duodenal ligament (Figure 8).

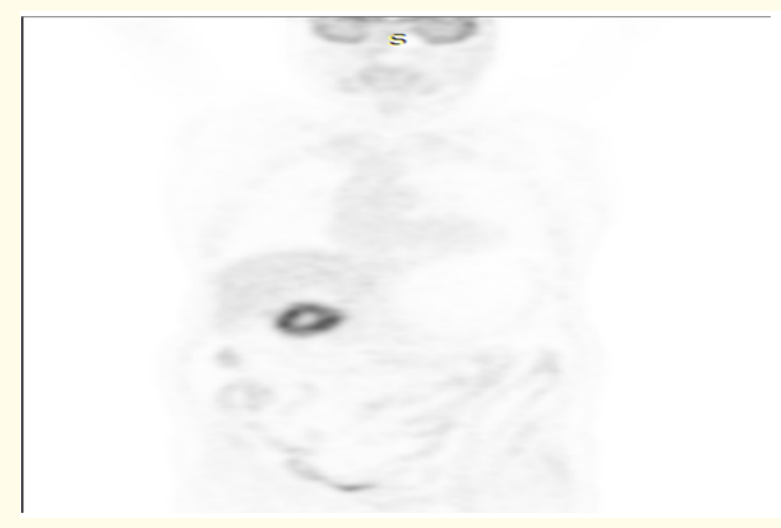

Figure 8: PET / CT of the body before surgery.

On April 9, 2019, an operation was performed: extended combined distal resection of the stomach with resection of the duodenal bulb with lymphodissection in volume D2. Postoperative pathohistology: low-grade adenocarcinoma, infiltrating all layers of the stomach wall, growing into the small omentum. with the presence of lymphovascular, venous and perineural invasion. Carcinoma metastases were found in 12 of the 27 lymph nodes. Next, 4 adjuvant chemotherapy was performed as a stage of perioperative chemotherapeutic treatment in the mode of Fluorouracil $2600 \mathrm{mg} / \mathrm{m}^{2} 24$ hour infusion on day $1+$ oxaliplatin $85 \mathrm{mg} / \mathrm{m}^{2}$ on day $1+$ calcium folinate $200 \mathrm{mg} / \mathrm{m}^{2}$ on day $1+$ docetaxel $50 \mathrm{mg} / \mathrm{m}^{2}$ on day 1 ; a cycle of 14 days. However, a CT scan of the OBP from 02.08 .19 found a compaction of fiber around the abdominal trunk, the common hepatic artery with the presence of a pathological component with a density of +26 units. $\mathrm{H}$, around numerous rounded lymph nodes up to $9 \mathrm{~mm}$. Immunohistochemistry from the central pathomorphological laboratory of St. Petersburg. Tumor cells are positive for MSH6, negative for PMS and MLH1. Conclusion: adenocarcinoma with signs of dMMR/MSI-H. Passed a PET/CT scan of the body on 15.10.2019. Conclusion: increased metabolic activity of FDG along the course of the colon, active metastases to single mesenteric and perinatal lymph nodes. According to fibrocolonoscopy data from 28.10.2019, no tumor lesion of the colon was detected (Figure 9).

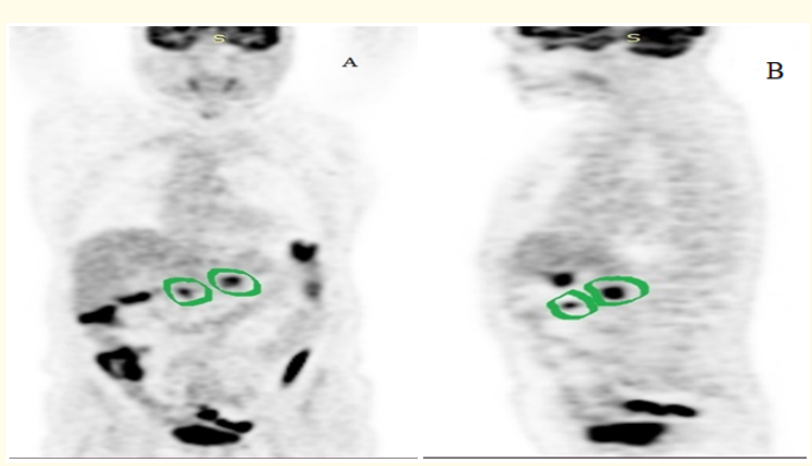

Figure 9A and 9B: PET/CT of the body 3.5 months after combined treatment. Enlarged retroperitoneal lymph nodes are outlined in green.

An oncological consultation was held. It was decided to carry out treatment in the mode of pembrolizumab $200 \mathrm{mg}$ on the $1^{\text {st }}$ day, a cycle of 21 days. On November 25, 2019, she received 1 course of immunotherapy in the mode of pembrolizumab $200 \mathrm{mg}$ on the 1 st day, a cycle of 21 days. In total, she received 19 courses of this treatment. PET/CT of the body from 09.04.2020 - Partial response. Regression of size and activity in retroperitoneal and mesenteric lymph nodes up to $12 \mathrm{~mm}$ SUVmax2, 2 (previously up to $31 \mathrm{~mm}$ SUVmax 12.3) (Figure 10).

PET/CT of the body from 04.09.2020-without negative dynamics. Stabilization of the disease. lymph nodes of the abdominal cavity and retroperitoneal space with sizes up to $12 \mathrm{~mm}$ of background activity of FDG. PET/CT of the body from 12.01.2021. Diffusemoderate activity of FDG in the walls of the stomach SUV max 2.9 (previously SUV max 3.09). Lymph nodes of the abdominal cavity and retroperitoneal space with sizes up to $12 \mathrm{~mm}$ with background activity of FDG SUV max 1.8. Conclusion: without negative dynamics (Figure 11). 


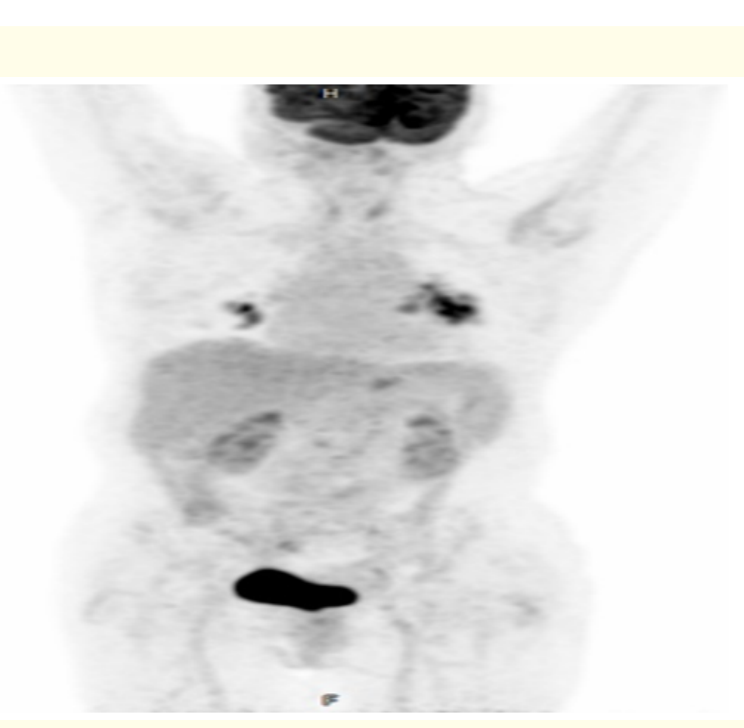

Figure 10: PET / CT of the body from 09.04.2020.

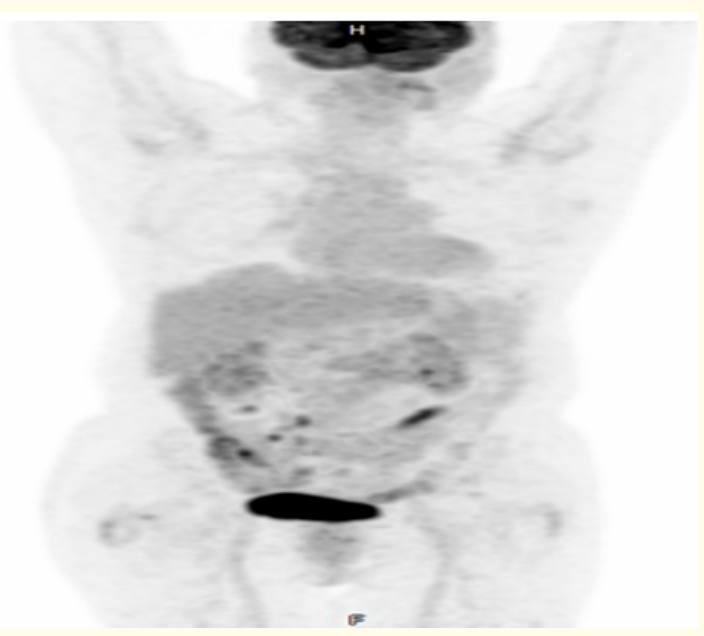

Figure 11: PET/CT of the body from 12.01.2021.

On March 2, 2021, the $1^{\text {st }}$ course of therapy with checkpoint inhibitors according to the scheme pembrolizumab $400 \mathrm{mg}$ on the first day, a cycle of 42 days was carried out.

She continued taking pembrolizumab in the above-described mode. On the next PET/CT scan of the body, it was revealed. Weak metabolic activity of FDG in the walls of the stomach SUVmax2, 8. Retroperitoneal lymph nodes up to 8 - $9 \mathrm{~mm}$ in size, with back- ground metabolic activity of FDG SUVmax1, 7. Stabilization of the disease.

She takes pembrolizumab $400 \mathrm{mg}$ once every 42 days. The following changes were found on the last PET/CT scan of the body from the end of August 2021. Diffusely weak metabolic activity of FDG SUVmax2,2 (previously up to SUVmax2,8) remains in the walls of the stomach. At the level of the epigastrium, in the loop of the small intestine, there is a focal hyperfixation of FDG, with metabolic dimensions up to $23 \times 31 \mathrm{~mm}$, SUVmax12. 06, without obvious structural changes on the native CT. Retroperitoneal lymph nodes up to $12 \mathrm{~mm}$ in size (previously up to $9 \mathrm{~mm}$ ), with background metabolic activity of ADH SUVmax2. 1(previously up to SUVmax1.7). It was regarded as the quality of stabilization of the disease (Figure 12).

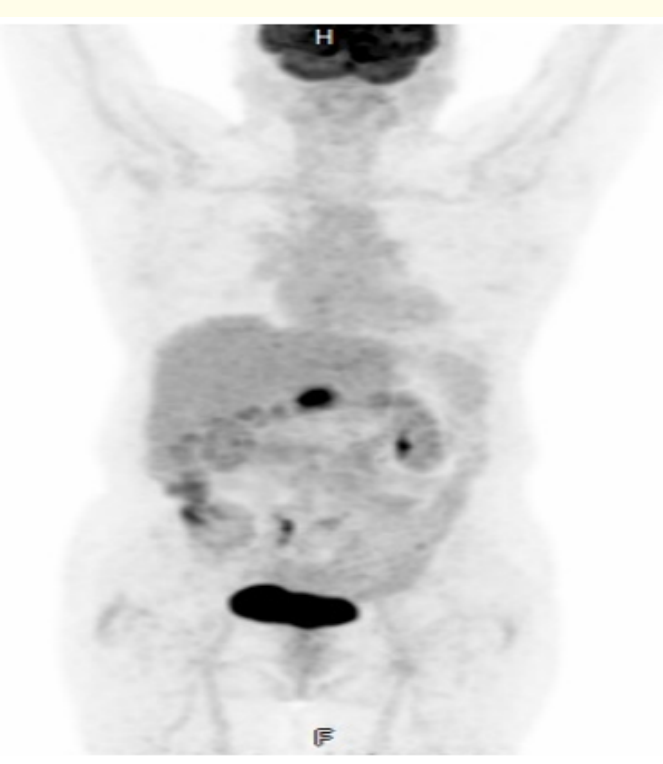

Figure 12: PET/CT of the body from 23.08.2021.

In total, she received 25 courses of immunotherapy with pembrolizumab.

\section{Discussion}

The patient has not had a recurrence of stomach cancer since November 2019 to the present. Progression-free survival was not achieved within 1 year and 9 months. 
According to the clinical study of the third phase of KEYNOTE-062, there were no static differences with chemotherapy in the overall survival in patients with metastatic gastric cancer using pembrolizumab.

The Phase IIb clinical trial KEYNOTE-659 investigated the use of pembrolizumab in combination with s-1 with oxaliplatin in the treatment of advanced gastric cancer in the first line. The overall response rate was $72.2 \%$. The relapse-free survival was 9.4 months, the overall survival was not achieved during the follow-up period. Adverse events of the $3^{\text {rd }}$ degree were observed in the form of thrombocytopenia, neutropenia, colitis, adrenal insufficiency.

Another phase II clinical trial, KEYNOTE-059, studied the use of pembrolizumab in previously treated patients with advanced gastric cancer and cancer of the cardioesophageal junction. Objective response rate was $11.6 \%$. The full answer was $2.3 \%$. The maximum response period to treatment was 17.3 months in patients with PDL1 positive status.

Given such a good partial response of stomach cancer to treatment with pembrolizumab and the duration of the lasting response of more than 21 months, it is possible to judge the effectiveness of the use of pembrolizumab in patients with advanced stomach cancer. We believe that the use of immunotherapy in super selective patients with stomach cancer can help in prolonging their life and improving their quality of life.

\section{Conclusion}

Despite the poor prognosis of the life of patients with stomach cancer, the study of the biology of stomach tumors in the form of medical and genetic analyses allows us to find new targets for the treatment of such an aggressive disease. The use of a monoclonal antibody, PD-1 inhibitor of pembrolizumab is an additional option for drug antitumor therapy for advanced stomach cancer. However, the presence of a mechanism of resistance to PD-1, PD-L1 inhibitors limits the widespread use of pembrolizumab and nivolumab in gastric cancer. The only biomarkers are MSI, PD-L1, but they do not guarantee effective treatment in specific individuals. Further study of checkpoint inhibitors, and the search for ways to overcome resistance to immunopreparations can give a new impetus to improving the results of treatment of stomach cancer.

\section{Bibliography}

1. M Ayers., et al. "IFN-gamma-related mRNA profile predicts clinical response to PD-1 blockade". Journal of Clinical Investigation 127 (2017): 2930-2940.

2. C Boger., et al. "PD-L1 is an independent prognostic predictor in gastric cancer of Western patients". Oncotarget 7 (2016): 24269-24283.

3. H Chang., et al. "Programmed death-ligand 1 expression in gastric adenocarcinoma is a poor prognostic factor in a high CD8+ tumor infiltrating lymphocytes group". Oncotarget 7 (2016): 80426-80434.

4. R Cristescu., et al. "Molecular analysis of gastric cancer identifies subtypes associated with distinct clinical outcomes". Nature Medicine 21 (2015): 449-456.

5. L Gu., et al. "PD-L1 and gastric cancer prognosis: a systematic review and meta-analysis". PLoS One 12 (2017): e0182692.

6. CG Jiang., et al. "Clinicopathologic characteristics and prognosis of signet ring cell carcinoma of the stomach: results from a Chinese mono-institutional study". Journal of Surgical Oncology 103 (2011): 700-703.

7. S Jin., et al. "The PD-1, PD-L1 expression and CD3+ T cell infiltration in relation to outcome in advanced gastric signet-ring cell carcinoma, representing a potential biomarker for immunotherapy". Oncotarget 8 (2017): 38850-38862.

8. A Kawazoe., et al. "Clinicopathological features of programmed death ligand 1 expression with tumor-infiltrating lymphocyte, mismatch repair, and Epstein-Barr virus status in a large cohort of gastric cancer patients". Gastric Cancer 20.3 (2017): 407-415.

9. K Yuza., et al. "Hypermutation and microsatellite instability in gastrointestinal cancers". Oncotarget 8.67 (2017): 112103112115.

10. ST Kim., et al. "Comprehensive molecular characterization of clinical responses to PD-1 inhibition in metastatic gastric cancer". Nature Medicine 24 (2018): 1449-1458.

11. J Lee and K Kim. "Biomarkers for gastric cancer: molecular classification revisited". Precision and Future Medicine 1 (2017): 59-68.

12. S Lemery., et al. "First FDA approval agnostic of cancer site when a biomarker defines the indication". The New England Journal of Medicine 377 (2017): 1409-1412. 
13. YX Liu., et al. "Prognostic significance of PD-L1 expression in patients with gastric cancer in East Asia: a meta-analysis". Oncology and Therapy 9 (2016): 2649-2654.

14. K Yuza., et al. "Hypermutation and microsatellite instability in gastrointestinal cancers". Oncotarget 8 (2017): 112103112115.

15. X Liua., et al. "High PD-L1 expression in gastric cancer (GC) patients and correlation with molecular features". Pathology Research and Practice 216 (2020): 1-7.

16. Salem ME., et al. "Landscape of tumor mutation load, mismatch repair deficiency, and PD-L1 expression in a large patient cohort of gastrointestinal cancers". Molecular Cancer Research 16 (2018): 805-812.

17. Bonneville R., et al. "Landscape of microsatellite instability across 39 cancer types". JCO Precision Oncology - ASCO Journals (2017).

18. Nakamura Y., et al. "Large-scale analyses of tumor mutation burdens (TMBs) across various advanced gastrointestinal (GI) malignancies in the nationwide cancer genome screening project SCRUM-Japan GI-SCREEN". Journal of Clinical Oncology 36 (2018): 12094.

19. Liu Y., et al. "Comparative molecular analysis of gastrointestinal adenocarcinomas". Cancer Cell 33 (2018): 721-735.

20. Network CGAR. "Comprehensive molecular characterization of gastric adenocarcinoma”. Nature 513 (2014): 202-209.

21. Y Eso., et al. "Microsatellite instability and immune checkpoint inhibitors: toward precision medicine against gastrointestinal and hepatobiliary cancers". The Journal of Gastroenterology 55 (2020): 15-26.

22. Chalmers ZR., et al. "Analysis of 100,000 human cancer genomes reveals the landscape of tumor mutational burden". Genome Medicine 9 (2017): 34.

23. Kang YK., et al. "Nivolumab in patients with advanced gastric or gastrooesophageal junction cancer refractory to, or intolerant of, at least two previous chemotherapy regimens (ONO4538-12, ATTRACTION-2): A randomised, double-blind, placebo-controlled, phase 3 trial". Lancet 390 (2017): 2461-2471.
24. Boku N., et al. "A phase 3 study of nivolumab (Nivo) in previously treated advanced gastric or gastroesophageal junction (G/GEJ) cancer: Updated results and subset analysis by PD-L1expression (ATTRACTION-02)". Annals of Oncology 28.5 (2017): 6170A.

25. BA Weinberg., et al. "Immuno-Oncology Biomarkers for Gastric and Gastroesophageal Junction Adenocarcinoma: Why PD-L1 Testing May Not Be Enough". The Oncologist 23 (2018): $1-7$.

26. Muro K., et al. "Pembrolizumab for patients with PD-L1-positive advanced gastric cancer (KEYNOTE-012): a multicentre, open-label, phase 1b trial". Lancet Oncology 17.6 (2016): 717726.

27. Kim ST., et al. "Comprehensive molecular characterization of clinical responses to PD-1 inhibition in metastatic gastric cancer". Nature Medicine 24.9. (2018): 1449-1458.

28. Le DT., et al. "PD-1 blockade in tumors with mismatch-repair deficiency". The New England Journal of Medicine 372.26 (2015): 2509-2520.

29. Sundar R., et al. "Transcriptional analysis of immune genes in Epstein-Barr virus-associated gastric cancer and association with clinical outcomes". Gastric Cancer 21.6 (2018): 10641070.

30. R Sundar., et al. "Epigenomic promoter alterations predict for benefit from immune checkpoint inhibition in metastatic gastric cancer". Annals of Oncology 30 (2019): 424-430.

31. SV Menghani., et al. "Gastric cardia adenocarcinoma with metastasis to the scalp: a case report". Cureus 12.1 (2020).

32. G Brar and MA Shah. "The role of pembrolizumab in the treatment of PD-L1 expressing gastric and gastroesophageal junction adenocarcinoma". Therapeutic Advances in Gastroenterology 12 (2019): 1-12.

33. SS Joshi., et al. "Pembrolizumab for treatment of advanced gastric and gastroesophageal junction adenocarcinoma”. Future Oncology 14. 5 (2018): 417-430.

34. I Chau., et al. "Ramucirumab in Combination with Pembrolizumab in Treatment-Naïve Advanced Gastric or GEJ Adenocarcinoma: Safety and Antitumor Activity from the Phase 1a/b JVDF Trial". Cancers 2985.12 (2020): 1-16. 
35. Ohtsu A., et al. "Pembrolizumab (MK-3475) versus paclitaxel as second-line therapy for advanced gastric or gastroesophageal junction (GEJ) adenocarcinoma: Phase III KEYNOTE-061 study". Journal of Clinical Oncology 34.4 (2016): TPS183TPS183.

36. Doi T., et al. "KEYNOTE-181: Phase 3, open-label study of second-line pembrolizumab vs single-agent chemotherapy in patients with advanced/metastatic esophageal adenocarcinoma". Journal of Clinical Oncology 34.15 (2016): TPS4140TPS4140.

37. Bang YJ., et al. "First-in-human Phase 1 study of margetuximab (MGAH22), an Fc-modified chimeric monoclonal antibody, in patients with HER2-positive advanced solid tumors". Annals of Oncology 28.4 (2017): 855-861.

38. Catenacci DVT., et al. "A Phase 1b/2, open label, dose-escalation study of margetuximab (M) in combination with pembrolizumab (P) in patients with relapsed/refractory advanced HER2+ gastroesophageal (GEJ) junction or gastric (G) cancer". Journal of Clinical Oncology 35.4 (2017): TPS219-TPS219.

39. Shah MA., et al. "Pembrolizumab (MK-3475) for previously treated metastatic adenocarcinoma or squamous cell carcinoma of the esophagus: Phase II KEYNOTE-180 study". Journal of Clinical Oncology 34.4 (2016): TPS189-TPS189.

40. Deng L., et al. "Irradiation and anti-PD-L1 treatment synergistically promote antitumor immunity in mice". Journal of Clinical Investigation 124.2 (2014): 687-695.

41. Chao J., et al. "Combining pembrolizumab and palliative radiotherapy in gastroesophageal cancer to enhance anti-tumor T-cell response and augment the abscopal effect". Journal of Clinical Oncology 35.4 (2017): TPS220-TPS220.

42. Phase $1 \mathrm{~b}$ open-label study of PEGPH20 with pembrolizumab.

43. Safety and efficacy of CRS-207 with pembrolizumab in gastric, gastroesophageal junction or esophageal cancers.

44. Rimm DL., et al. "A prospective, multi-institutional, pathologist-based assessment of 4 immunohistochemistry assays for PD-L1 expression in non-small cell lung cancer". JAMA Oncology 3.8 (2017): 1051-1058.

45. Tran PN., et al. "PD-1 and PD-L1 as emerging therapeutic targets in gastric cancer: current evidence". Gastrointestinal Cancer 7 (2017): 1-11.
46. Farris AB 3rd., et al. "Clinicopathologic and molecular profiles of microsatellite unstable Barrett Esophagus-associated adenocarcinoma". The American Journal of Surgical Pathology 35.5 (2011): 647-655.

47. Zhu L., et al. "Microsatellite instability and survival in gastric cancer: a systematic review and meta-analysis". Molecular and Clinical Oncology 3.3 (2015): 699-705.

48. Smyth EC., et al. "Mismatch repair deficiency, microsatellite instability, and survival: an exploratory analysis of the Medical Research Council Adjuvant Gastric Infusional Chemotherapy (MAGIC) Trial". JAMA Oncology 3.9 (2017): 1197-1203.

49. Gryfe R., et al. "Tumor microsatellite instability and clinical outcome in young patients with colorectal cancer". The New England Journal of Medicine 342.2 (2000): 69-77.

50. Dudley JC., et al. "Microsatellite instability as a biomarker for PD-1 blockade". Clinical Cancer Research 22.4 (2016): 813820.

\section{Volume 3 Issue 10 October 2021} (C) All rights are reserved by Rakhimov $\mathrm{R}$ Radmir., et al. 